\title{
Certification of Unmanned Aircraft Systems - from product safety to type certificate - a review about the operation of the EU safeguard processes
}

\author{
Zs. Sándor ${ }^{1, *}$, M. Pusztai ${ }^{1}$ \\ ${ }^{1}$ KTI Institute for Transport Sciences Non-profit Ltd. \\ Than Károly street 3-5, H-1119 Budapest, Hungary \\ *e-mail: sandor.zsolt@kti.hu
}

Submitted: 09/08/2021 Accepted: 04/10/2021 Published online: 18/10/2021

\begin{abstract}
Significant changes are emerging in the market of unmanned aircraft systems since 2019 through the publication of two specific regulations that regulate all steps of the use of unmanned aerial vehicles in detail. With the implementation of the new EU drone regulations, the role of the notified bodies and the certification agencies will be more important from the viewpoint of product safety and the official certification required by the EU and national aviation authorities. The product safety chain consists of two major parts. One part belongs to the production phase, where the manufacturer has to prove the functionality (it is called the conformity assessment) and another part belongs to the distribution market, where the authorities assess the fulfilment of the conditions of the distribution (it is called the market surveillance). The first pillar concern to the design and manufacturing and the second to the distribution. Each segment is presented in this article and the authors introduce the different control approaches of these segments. It has to be taken into consideration that the drones are representing a special market with notable safety risks that have to be handled during the whole life path of the products from the design through the distribution until the aerial operations.
\end{abstract}

Keywords: certification; unmanned aircraft systems; product safety; safeguard; conformity assessment; market surveillance

\section{INTRODUCTION}

Unmanned Aerial Vehicles (UAVs) play a significant role in the industry of Europe. With this equipment, several human-related resources can be substituted. The global market has already identified it, thus the industry of drones is prospering. In 2020 the market of UAVs was valued at 30601.14 million USD, and researchers projected its compound annual growth rate to $8.5 \%$ for a 5 year forecast period between 2021 and 2026 [1].

Europe has a prominent position in this market. The European unmanned aerial vehicle market accounted for 2637.2 million USD in 2019 and will grow at $\sim 10 \%$ annually between 2020 and 2026 . Within 20 years, the European drone sector is expected to directly employ more than 100,000 people and it will have an economic impact exceeding 10 billion EUR per year, mainly in services [2].

These numbers are justifying the fact that rule makers and national decision-makers should handle the topic and the global European approach should be developed from the design through the manufacture until the use of these products.

According to the Treaty on the Functioning of the European Union, member states and EU institutions shall take all measures necessary for the creation of the single market where borders shall mean no obstacle to the free movement of products (i.e., goods) [3]. To this aim, it is vital, however, that products moving without barriers are safe.

Due to technical development, many products, that are easy to access but are very complicated and pose risks even at ordinary use, appeared in everyday lives. For an average person - called consumer (the term of the European law) - cannot be even legally expected the understanding of these products' technical background. The legislator shall be satisfied once the consumer undertakes the burden of learning the ways of the product's normal use and the inherent risks thereof (i.e., reading the user's manual). Every other task shall be fulfilled by the manufacturer or by other players in the supply chain 
(e.g., the importer and/or the distributor). This applies to all branches of the industry as well as to Unmanned Aircraft Systems (UAS) that constitute the topic of the article.

These circumstances called the product safety rules to life, which - through the (EU) 2019/945 Delegated Regulation (hereinafter DR) of the European Commission - created specific provisions in connection with UASs [4].

To provide fulfilling these tasks by the manufacturers properly and that level of product safety is sufficient in case of UAS intended to be operated in "open" category as provided for in Commission Implementing Regulation (EU) 2019/947 (hereinafter IR), the European legislator simultaneously with the supply chain - created the product safety chain, including roles and competencies therein [5]. Players of the product safety chain are available in adequate phases of the supply chain and are capable of intervening in precisely defined stages. The two most important actors of the product safety chain are the conformity assessment bodies (i. e., NOtified BOdy, hereinafter NOBO) and authority for market surveillance.

The concept of product safety chain does not appear in literature and relevant laws. The other players of the supply chain (manufacturer, importer, distributor) have some obligations in connection with product safety however, these obligations are not characterizing. Meanwhile, the two pillars of the product safety chain (conformity assessment and market surveillance) deal only with product safety, and thanks to their strict regulations they are completely independent of every other actor of the supply chain. The concept of product safety chain has been elaborated to envisage the different roles and competencies and the legal, organizational, and economic independence and separation. It constitutes another ground for this separation that the two pillars of the product safety chain make an external audit of the supply chain in different stages of products therein.

In "specific" and "certified" operational categories as provided for in IR, the European legislator went further and stepped over the logic of product safety, and is approaching the regulation on certification of regular aircraft in terms of regulatory structure and mind-set.

In the present article, the authors are intending to show the legislative solutions of each operational category and the regulatory "arch" linking them together into a single system. The product safety chain consists of two pillars. The conformity assessment is presented in Section II and the market surveillance, which is the second pillar is presented in Section III. The operations of UASs are regularized in detail but until the product safety chain is not operating completely due to the lack of legislative gaps, some transitional provisions are in place. Section IV. deals with these provisions. Section V. presents the operational issues, while Section VI. gives a summary of the conventional certification process connected to UASs.

\section{THE CONFORMITY ASSESSMENT - THE FIRST PILLAR OF THE PRODUCT SAFETY CHAIN}

Air transport is a dangerous operation field where safety plays a key role. This safety might be achieved in different ways: training of experts in the industry, use of generally accepted directives/standards/regulatory means, and control of equipment throughout the whole cycle of their life from design to disassembly.

In case of UASs intended to be used in the "open" operational category as provided for in IR, the legislator warrants safety via conformity assessment of UAS, which is the first step of product safety and focuses on design, organization of production, and control of the manufacturer's activity thereby defending consumers from risks.

The product safety and market surveillance approach imply that the Delegated Act (DA) considers UAS with "CE marking" - Certificate of Compliance - and class identification label primarily as consumer goods. The "CE marking" is indeed a certificate of compliance, but it is not the abbreviation of "certificate of compliance". It is a unique marking which should proof that the product complies with defined standards of the European Economic Area. To summarize, as these products are aerial vehicles at the same time, it is possible to define them as consumer goods that are capable of flying.

It is important to set forth: this shall not mean that - in terms of flight safety - there would be any difference between drones operated in the "open" category or Standard Scenarios (hereinafter STS) as in IR and the unmanned aircraft operated in the stricter "specific" or "certified" operational categories [3]. On the contrary, the legislator achieves a sufficient level of air safety via product safety/consumer protection/market surveillance regulations in operational categories having lower risk (like the "open" category and STS).

There is another aspect behind choosing conformity assessment as a means of certification: the introduction of certification process applied for regular aircraft would result in disproportionately severe technical and financial burden on manufacturers' side in case of UAS having a Maximum Take-Off Weight (MTOW) of $25 \mathrm{~kg}$ and being used in a very limited operational environment. This could prevent many manufacturers from entering the market and could 
lead to a regression of the drone market in the long run.

Therefore, the DR and European Aviation Safety Agency (EASA) created a system, where UAS with MTOW no more than $25 \mathrm{~kg}$ - in case if they are targeted to be operated in the "open" category or STS as provided for in IR - are treated as products and the risk they pose by appearing in air traffic is handled with the introduction of UAS-specific product safety rules.

The first pillar is in this system is the conformity assessment, which as a regulatory technique is common since the 765/2008/EC Regulation that is completed by the $768 / 2008 / \mathrm{EC}$ Decision, that defines so-called conformity assessment modules. The conformity assessment has already been mandatory for drones before the DR, upon completely different standards and regulations, however [6], [7].

These modules are general and not limited to certain branches. When the European Union wishes to introduce conformity assessment on a certain new field of law as a regulatory means, however, it will define in the specific legal act - like DR - which conformity assessment modules it will make available in the specific branch. The novelty of the DR is that it constituted single and specifically UAStailored conformity assessment rules.

Concerning the topic of the present article, these modules shall be distinguished upon whether the NOBO is required during conformity assessment or the manufacturers themselves - undertaking all liability - can conduct the procedure, and the market surveillance authority shall supervise appropriateness of the assessment subsequently.

Conformity assessment might be performed according to the modules thereof, but these different modules are made available for actors of the supply chain in case of different sorts of products within the realm of UASs. The Annex of DR lists seven different Unmanned Aircraft Classes (UA Class) according to the technical requirements and capabilities thereof. The UA Class determines at the same time; which operational category the drone may fly. Table 1 shows the conformity assessment modules applicable to different UA Classes. It is clear from the chart that Module A is not an option for the manufacturer in the case of UA Classes $\mathrm{C} 1$ to $\mathrm{C} 3$ and the NOBO shall be part of the process in these cases.
Table 1. Modules in conformity assessment of unmanned aerial vehicles

\begin{tabular}{|c|c|c|c|}
\hline $\begin{array}{c}\text { Conformity } \\
\text { Assessment } \\
\text { Module }\end{array}$ & $\begin{array}{c}\text { Content of } \\
\text { the Module }\end{array}$ & UA Class & $\begin{array}{c}\text { Is a } \\
\text { Notified } \\
\text { Body } \\
\text { (NOBO) } \\
\text { required? }\end{array}$ \\
\hline A & $\begin{array}{c}\text { Internal } \\
\text { production } \\
\text { control }\end{array}$ & C0, C4-C6 & none \\
B, C & $\begin{array}{c}\text { EU-type } \\
\text { examination, } \\
\text { Conformity } \\
\text { to type } \\
\text { based on } \\
\text { internal } \\
\text { production } \\
\text { control }\end{array}$ & C0-C6 & yes \\
\hline $\mathrm{H}$ & $\begin{array}{c}\text { Conformity } \\
\text { based on full } \\
\text { quality } \\
\text { assurance }\end{array}$ & C0-C6 & yes \\
\hline
\end{tabular}

\section{Conformity assessment upon harmonized standards}

In the case of drones, Module $\mathrm{A}$, the internal product control might be chosen by the manufacturers to conduct conformity assessments on their own. This module applies exclusively to UA Class $\mathrm{C} 0$ and $\mathrm{C} 4$ to $\mathrm{C} 6$ at the same time. It would be applicable: the harmonized standards required for the proper conduct of the procedure have not been completed yet. These new standards will constitute the so-called EN 4709 standards and Table 2 lists the elements thereof [8].

It is clear from the naming that the DR introduced new technical requirements, so new drone types sold to operate in the "open" category or STS - shall meet these new requirements before being launched on the market.

The new harmonized standards are so important because the application thereof fastens the conformity assessment procedure and market surveillance. The DR provides the presumption of conformity to these standards, which means that - if the manufacturer refers thereto - it shall not be investigated during the conformity assessment procedure whether the manufacturer chose the adequate technical process to achieve conformity, but only the result i.e., the fulfilment of technical requirement prescribed by law (e.g., DR) shall be verified and checked. 
Table 2. The elements of EN 4709 standards

\begin{tabular}{|c|c|}
\hline $\begin{array}{c}\text { Name of the } \\
\text { (sub)standard }\end{array}$ & $\begin{array}{c}\text { Content of the } \\
\text { (sub)standard }\end{array}$ \\
\hline EN 4709-1 & $\begin{array}{c}\text { Harmonized standard on } \\
\text { product and verification } \\
\text { requirements for } \\
\text { Unmanned Aircraft } \\
\text { Systems intended to be } \\
\text { operated in the "Open" } \\
\text { category }\end{array}$ \\
\hline EN 4709-2 & $\begin{array}{c}\text { Harmonized standard on } \\
\text { direct remote } \\
\text { identification for } \\
\text { Unmanned Aircraft } \\
\text { Systems }\end{array}$ \\
\hline EN 4709-3 & $\begin{array}{c}\text { Harmonized standard on } \\
\text { geo-awareness for } \\
\text { Unmanned Aircraft } \\
\text { Systems }\end{array}$ \\
\hline EN 4709-4 & $\begin{array}{c}\text { Harmonized standard on } \\
\text { lights for Unmanned } \\
\text { Aircraft }\end{array}$ \\
\hline
\end{tabular}

\section{Conformity assessment with NOBO}

The DR provides exclusively $\mathrm{B}+\mathrm{C}$ Modules for UA Class C1 to C3. In Module B (EU-type examination), the intervention of $\mathrm{NOBO}$ is mandatory. In the frame of this module, the NOBO shall issue an EU-type examination certificate following the evaluation of the manufacturer's technical documentation and testing of already produced specimens. The manufacturer may only proceed with conformity assessment with Module C in possession of the EU-type examination certificate and later may commence the placing on the market of the drone.

It is the European Commission that grants the status of being notified body to NOBOs and records them, following the handover of documentation by the competent notifying authority (this is the Civil Aviation Authority (CAA) in Hungary). The EUtype examination certificate, issued by NOBO shall provide the opportunity of trading throughout the European Union.

The NOBOs may intervene in conformity assessment during the application of Module $\mathrm{H}$ too. In the case of this module, the NOBO performs full quality assurance and evaluates not the specific product but the production. Therefore, this is not a one-time but a regular inspection on the manufacturer's site.

Until now, no conformity assessment body was recorded on the NANDO (New Approach Notified and Designated Organisations) list of the European Commission where these bodies are publicly available [9]. Regarding that notification shall be preceded by accreditation and the process itself before the European Commission takes at least two months - if no notifying authority from member states questions the notification -, the appearance of NOBOs is yet to come.

It is another important circumstance for drones with UA Class C5 or C6 that operational rules for STS shall enter into effect only in 2023. This means, that these kinds of operations are not permitted before that day, even if UAS with C5 or C6 class identification label existed on the market.

The EASA realized the indefensibility of this situation (i.e., there is no UAS equipped with class identification label) and created PDRA-S01 and PDRA-S02 within the framework of Pre-Defined Risk Assessment (PDRA)-s, where - apart from the obligation of using drones with $\mathrm{C} 5$ or C6 class identification label - every circumstance is identical with the ones in STS operations. This way the acquisition of operational authorization is still required however, the process has been facilitated as the EASA had defined the basis of risk assessment in advance.

\section{Conformity assessment of privately built UAS}

In the course of establishing the IR, the European Commission so took into consideration that there were many vehicles in the European sky that had been custom-designed and -built by their operators. This further means that the technical conformity and airworthiness of these vehicles have never been controlled by an independent entity. Accordingly, the privately built UAS has no manufacturer but a builder, which further implies that the latter shall not undertake the obligation that a manufacturer does. This alleviation has its price at the same time: the IR introduced several restrictions on the operation of privately built UAS in the "open" category. These restrictions are the following:

- $\quad$ privately built UAS may only be operated in A1 subcategory (MTOW $<0.25 \mathrm{~kg}$ ) or in A3 subcategory (MTOW < $25 \mathrm{~kg}$ ); and

- $\quad$ exclusively for the builder's use.

Especially, the latter provision that can be derived from the concept of privately built UAS is interesting and means that such UAS is not marketable, it may not be sold, otherwise the term "for the builder's use" would lose its meaning.

These restrictions limit the operational opportunities of these privately built vehicles substantially, but at least the performance of certain operations remains feasible.

The abovementioned limitations apply to operations in the "open" category, but there is no 
legal obstacle to the use of privately built UAS in the "Specific" category. In the latter case, the process in Paragraph 4 of the present article is applicable, the technical conformity and airworthiness of these UASs shall be determined by the risk-mitigating measures defined in the operational authorization or Light UAS operator's Certificate (LUC) issued by the competent CAA. Presumably, competent CAAs will prescribe many tests and certification obligations before issuing operation authorization or LUC.

\section{MARKET SURVEILLANCE AUTHORITY AS THE SECOND PILLAR OF THE PRODUCT SAFETY CHAIN}

As the second pillar of product safety, the market surveillance is a bigger unit, which means a formaland content-based assessment, similar to the conformity assessment (see especially Module B). In the literature of the European Union, the reference for market surveillance is indicated as a compliance assessment [10]. There is a difference between the compliance and the conformity assessment, but both pillars contain assessment procedures that indicate the close relationship between the two pillars.

According to the fact that the first unit of the product safety chain (the conformity assessment, as described in the second paragraph of this article) is not yet able to fulfill its function, the market surveillance authorities have even more significant roles until the appearance of the new standards and the NOBOs.

Although the IR applies only to drones belonging to the UA Class C0-C6 that already went through the conformity assessment, this does not mean that the market surveillance authorities would not have any duty until the appearance of new drones.

According to the fact described in Section II.2 of this article, currently, all drones, which are being placed on the market or made available thereon with a class identification label, got these identification labels illegally and they might mislead the consumers. Thus, it may purport that the given drone can be legally used for the UAS operations according to the class identification label.

However, it is not true, because the IR limits the use of drones that do not have a class identification label in the "open" category, and the A2 subcategory is excluded from the available possibilities. (Article 20 and 22 of the IR. It has to be noted that this limitation is not absolute. If the UAS operator has an operational authorization, it is possible to execute an operation that has the characteristics of the A2 subcategory operation with a UAS that does not have a class identification label.)

Thus, the market surveillance authority is entitled to conduct inspections and audits, not only at the manufacturers' but also at the importers', distributors', and the dealers' sites. In case of infringement, the market surveillance authority is entitled to apply the whole Hungarian and EU toolkit of market surveillance laws, which contains even the recall of the product from the entire EU market besides the infliction of fines and penalties.

The market surveillance will also have a significant role in the future because the manufacturers shall keep the technical and compliance assessment documents of the drone that already went through the conformity assessment. These documents should be presented upon request of the national market surveillance authority for 10 years after the product has been placed on the Union market.

It is another important provision that the manufacturer of a drone with a C5 or a C6 class identification label should notify the market surveillance authority about any new type in these UA Classes before placing the product on the market. The market surveillance authority is entitled to ask for documents from the NOBOs about the conformity assessments of drones done by NOBOs. According to relevant legal regulations, it can be stated that the NOBOs and the market surveillance authority constitute one single system. As part of the product safety chain, they are operating jointly, not isolated. This operating method validates the use of the pillar metaphor.

Let us take an exaggerated, but still very clear example: while the conformity assessment functions as a sort of type-certification, the market surveillance should monitor the continuous airworthiness by the use of test purchase and labour tests. These provide additional external control in the further phase of the product safety and supply chain during the conformity assessments.

This is especially important in the case of Module A, where the manufacturer conducts the assessment individually. The EU would execute these assessments in an institutional form in the latter, by the establishment of union test sites [11]. These assessments with these sites would strengthen the industry and raise it above the level of the Member States, thereby creating a single internal market.

\section{TRANSITIONAL PROVISIONS OF THE IMPLEMENTING REGULATION}

The legal and organizational (more correctly the standardization) background of the conformity assessment is not yet available. To handle these situations and to provide a sufficient preparation period, the IR has defined certain transitional provisions. It is important to emphasize, that these transitional provisions are concerning the operations of the „open” category only. In case of operational 
authorization or with a LUC the UAS operator may use its UAS differently from these rules.

According to Article 20 of the IR following its entry into force, but not later than 31 st December 2022 , it is possible to place UASs on the market that is intended to be operated in the "open" category and are not in compliance with all requirements of the DR - consequently do not have a Class Identification Label (CIL).

As provided for in the IR, the operational possibilities of these UASs are limited in the "open" category too. Without CIL, the A1 subcategory is available only to new UASs with MTOW less than 500 grams, provided that the member state has elaborated competence-requirements (other than UAS.OPEN.020) to these operations (As Hungary created no such requirements, only drones under 250 grams can be operated in A1 subcategory without CIL in Hungary.). UASs newly placed on the market with MTOW at least 500 grams but less than $2 \mathrm{~kg}$ can be used only in subcategory A2 without CIL, provided that the remote pilot has a competence equivalent to the one in UAS.OPEN.030 [5] and the drone maintains a minimum of 50-meter distance from other people.

UAS having MTOW of at least $2 \mathrm{~kg}$ but under 25 $\mathrm{kg}$ can be operated in the A3 subcategory without a class identification label. Once the above transitional period is over only those UAS can be operated without CIL in the "open" category (A1 with MTOW under 250 grams and A3 with MTOW under $25 \mathrm{~kg}$ ) that has been placed on the market before 1st January 2023. UAS operators may differ from the provisions only in the possession of an operational authorization or a LUC.

UAS without a class identification label may be placed on the market after the 1st January 2023 as a new type too, but it can be operated only in the "special" category, thus an operational authorization or a LUC will be necessary for the fulfilment of the operations. Fig. 1 will show the transitional period and its relevant deadlines [12].

It means that considering these dates the actors of the supply chain should inform the buyers about the operational limits regarding the operations. The fulfillment of the information shall be inspected according to Article 39 of the DR by the market surveillance authorities of the Member States.

The situation is complicated because the IR defines deadlines to the act of placing on the market, not to act of making available on the market (Placing on the market means the first making available of a product on the Union market - according to the IR). If placing on the market is performed until the indicated deadline, the product can be distributed in the EU, until the manufactured stock runs out, without any obligation to acquire an operational authorization or a LUC for the operation of the given products.

Based on the prognosis of the EASA the potential buyers will be able to buy UASs for "open" category operations without class identification labels due to the fact these kinds of products will be available until 2026 at the merchants [13].

The manufacturers should not have to start the planning of the products from the beginning to

\section{Transitional periods connected to the use of UAS with or without class identification labels (CIL)}

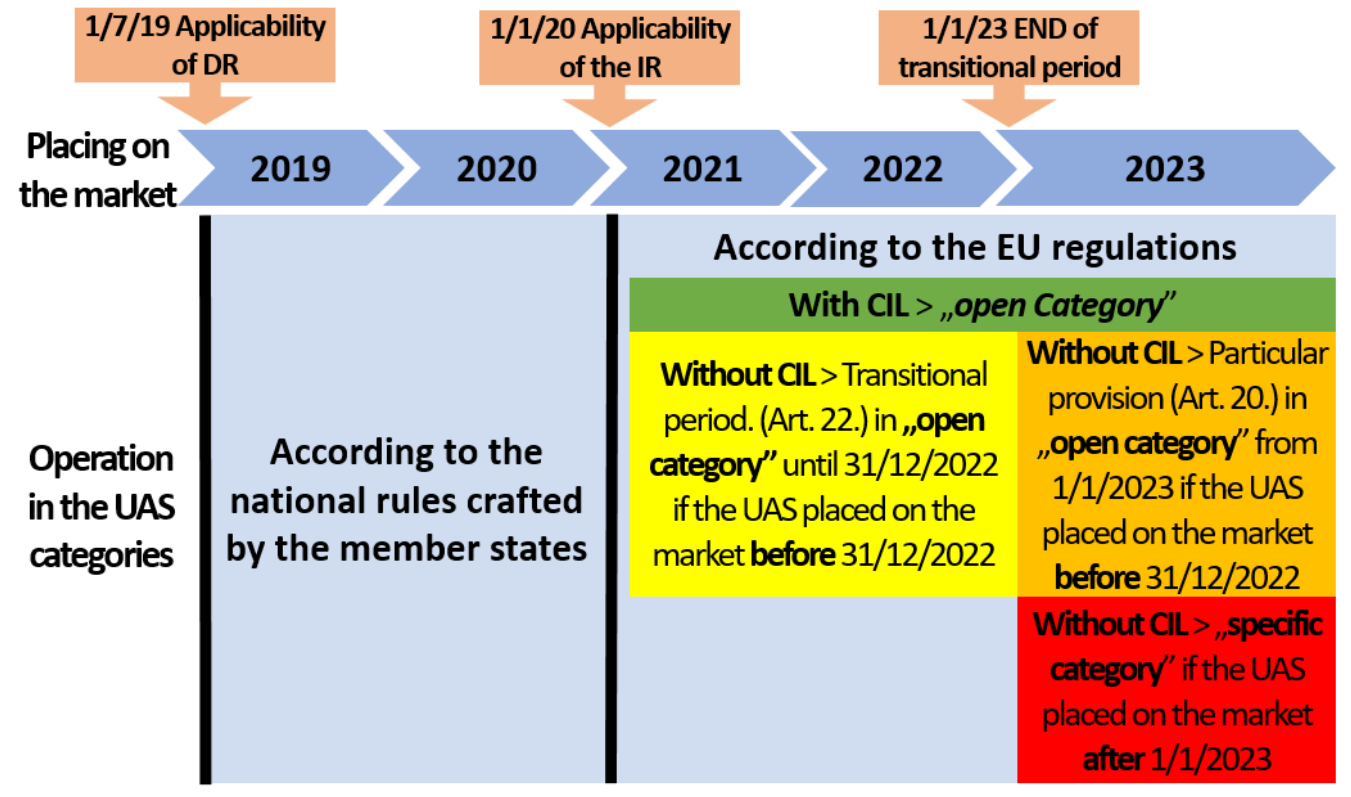

Figure 1. Transitional period for the use of UAS without CIL 
comply with the rules and regulations. If a model is fully compliant with the new rules and regulations after an upgrade or a retrofit, then the retrofit or the upgrade will be the basis of the conformity assessment. According to the regulations, the upgraded or retrofitted models are new models, thus they will be granted with new serial numbers irrespective of the scale of the modification.

Market surveillance authorities will have a significant role in the additional inspections of the new products that appear in the market, because according to the plans of the EASA, the operator may place the new identification labels on the UAS following the manufacturer's instructions after the controlled upgrade [13]. The administrational background of these inspections could be facilitated if the registration of the UASs were compulsory. This process can track the lifecycle of each UAS. Almost only Hungary has this kind of registration in the EU (the registration has a wider scope in France than the minimum requirement of the EU).

It is not obvious at the same time, why the European legislator considers drones without class identification label as meaning lower hazard to flight safety during the transitional period than after it. This applies especially to transitional limitations in subcategory A2, where the limitations (in terms of MTOW and distance) are supposed to balance the risk posed by the lack of conformity to DR and CIL. This provision outlined in Article 20 and 22 of the IR might serve as a legislative means to force manufacturers to provide upgrade or retrofit opportunities to operators of elder UASs - or to make UAS-operators buy new drones.

\section{THE AUTHORIZATION OF THE OPERATIONS IN THE "SPECIFIC" CATEGORY - RISK ASSESSMENT AND DESIGN VERIFICATION REPORT}

\section{Risk assessment}

The UAS operator shall acquire an operational authorization if the intended operation may not be executed in the "open" category or according to STS. The UAS operator shall submit a detailed risk assessment to the competent authority (except if the operator executes the operations based on a PDRA) [5]. It is the requirement of the acquisition of the operational authorization.

In the course of risk assessment, the Specific Assurance and Integrity Level (SAIL) is determined based on the environmental characteristics of the operational area (characteristics of the UAS, location of the operational area, airspace characteristics above the operational area, etc.). SAIL comprises levels from I to VI and a given level defines the degree of the risk of a certain operation in a complex way. The SAIL levels identify to which level the robustness of the given Operational Safety Objectives (OSOs) shall be assured in a specific operation [14].

The OSOs are the sum of all risk mitigation activities and safety barriers, which provide the mitigation of the effects of possible risks that might influence the operation. So far, 24 OSOs have been defined by the EASA. They concern the specific areas of the UAS operations (design of the UAS, maintenance, operation, competencies of the remote pilots, human errors and factors, operations under adverse weather conditions, etc.) and determine the related criteria and/or requirements. Based on the risk characteristics of the given operation, the SAIL levels determine which robustness level (low, medium, high) has to be achieved connected to the operation. In the case of low risk, the fulfilment of some OSOs is optional [15].

The robustness is the feature of the risk mitigation measures, which consists of two factors:

- level of integrity and

- level of assurance.

Both factors have low, medium, and high levels. The combination of these factors results in robustness. The level of robustness will always be equal to the lowest level of either the integrity or the assurance.

The EASA defined certain OSOs that can be validated by an independent third party and also defined those, which can be validated only by them. In the case of some OSOs, the medium or high robustness levels require the design verification report by the EASA.

\section{The design of verification report}

EASA decided to divide operations into three groups based on their SAIL. For operations in SAIL I and II that are considered as low-risk operations, the EASA accepts if the UAS operator provides conformity declaration of the manufacturer (that is available in conformity assessment) and any further step (i.e., higher level of verification) to prove conformity is optional.

But in the case of SAIL III-IV operations, it is up to the National Aviation Authorities (NAAs) to decide whether design-related OSOs shall be proven by a process called design verification. This process is conducted by EASA that will - similarly to regular aircraft - investigate technical issues of the UAS, but only those, and the OSOs in connection with the production and every other OSO remain in the competence of NAA to evaluate the robustness of documentation provided by the UAS operator [14], [15], [16], [17]. 
The EASA will, therefore, investigate the compliance of the following elements:

- $\quad$ the full design of the UAS;

- the mitigation means linked with the design; and

- the enhanced containment function.

As said the decision whether design verification is required or not the NAA shall bear the liability for not prescribing the need for the above process of EASA. Therefore, the EASA recommends to NAAs to choose this option, especially in case of operations over populated areas (The concept of populated area is yet to be clearly defined by the European legislator). Another important feature of the design verification report is that it is strictly linked to the concept of operations provided by the UAS operator (this can be derived from the investigated mitigation measures that depend on the concrete operation).

This means that this report has no general effectiveness covering all possible future operations and shall not result in the acquisition of the operational authorization or LUC automatically. It neither means that all UAS, that possesses a design verification report can be used in all operations of the "specific" category without further action required.

The rest of OSOs, including the production-related ones, will remain in national competence that further questions the effectiveness of this procedure contrary to conformity assessment where design and production form one single unit. If it is considered, that the validity of the design verification report unlike type certificate - will be limited to the European Union, the sense of introducing a third model between conformity assessment and type certification becomes doubtful.
Not to mention, that Article 13 of the IR already provides mutual recognition for operational authorizations and LUCs that means that these documents can be acquired from one NAA with validity to the entire European Union, - if the NAA so decides - without design verification report of the EASA.

The fact, that the NAAs still have the right to decide about design-related OSOs, hinders the creation of uniform application of EU law and consequently the single European market in the field of unmanned aircraft systems, which runs counter to aims defined in the preamble of the IR. The following Fig. 2 shows the concept of EASA to the system of conformity, verification, and certification of UASs [16].

It is visible, that there is a shift in terms of regulatory mindset in the "specific" category from product safety towards the transport/flight safety approach. The essence of this change is the handling of the proven methods: the operation and the management of detected risks during the operation are in the focus of the design verification report.

Contrary to the UASs used in the "open" category, the UAS operator bears more responsibility for the compliance of the UAS as the determiner of the characteristics of the operations in the "specific" category, where the conformity means the capability of maintaining the high level of flight safety by mitigating the risks, especially in the field of design.

In the end, the operational authorization or LUC (either with or without design verification report) will - according to Article 40 par. (3) of the DR serve as a document to declare the conformity of the UAS with the operation it is intended to be used for.

\section{Verification of the design of the UAS}

\begin{tabular}{ll}
\multicolumn{1}{|c|}{ Low risk } \\
(SAIL I and II)
\end{tabular}

Figure 2. Regulation system of the UAS certification according to the EASA recommendation 


\section{TYPE CERTIFICATE - THE "CERTIFIED" CATEGORY OF UAS OPERATIONS}

The "specific" category acts as a "bridge" between the rules of the "open" and the "certified" category. A glaring example of this is the list of UAs operation-types that are subject to certification indicated in the DR. According to Article 40 (1) point (d) of the DR, if the risk mitigation is not possible without a certification - based on the risk assessment conducted by the aviation authority -, a "type" certification fulfilled by the EASA shall be compulsory for the given UAS, that is operated in the "specific" category.

The ex-lege "certified" category UAS operations are subject to EASA certification, although the certification regulations for UASs - optimized versions of the conventional aircraft certification rules - are currently not available. Only ideas are existing about these kinds of processes [18].

This is the reason, why the electric Vertical TakeOff and Landing (eVTOL) drone "air-taxi" - which is suitable for passenger transport and the bestknown form of a drone to be operated in the "classified" category - has not appeared and spread yet in daily traffic, although many developments are underway.

Fig. 2 shows, however, a very interesting novelty in the interpretation of Article 40 (1) d) of the DR.

According to the above Fig. 2, the EASA provides a greater legem interpretation to SAIL V and VI operations as making them falling under the above provision of the DR. By the acceptance of the above, far more "specific" category operations shall require type certificate as provided for in "Part 21", similarly to regular aircraft and this might lead to disproportionate burden within the "specific" category and vanish the border between "specific" and "certified" category (Part 21 of Annex I to Commission Regulation (EU) No 748/2012 of 3 August 2012 laying down implementing rules for the airworthiness and environmental certification of aircraft and related products, parts and appliances, as well as for the certification of design and production organizations) [19].

\section{OUTLOOK}

In the future until 2023 there will be limited number of UAS with CIL. It is limiting the legal possibilities of the flight missions and require more authorizations which need more human effort (due to the single operational authorization). In this period the transitional provisions of the IR ensure the possible operations with limitations In the next few years NOBOs will emerge in the field of UASs but the spread of these organizations will require years. New standards will be published and the EASA has started programs for the establishment of the technical regulations concerned to UAS.

The future works in the field of the topic will concern to the standards and the new technical functions and their assessment. UASs have a lot of new technical functions that should be correctly evaluate and the new standards will contain the methods. Research of the functional appropriateness of the new technical possibilities will be appreciated in the future. Manufacturers should use new evaluation methods with the involvement of NOBOs, and the assessment procedure will cover more functions that nowadays.

The emergence of CIL will facilitate the use of UAVs both in the industrial and the recreational segment because it will clearly define the usage possibilities and the necessary legal and technical requirements.

\section{Conclusions}

In the article, the authors have presented the regulatory system of the UAS certification and its connections. This system - according to the level of risk - starts with conformity assessment and through design verification report - leads towards "Part 21" certification. Special attention was dedicated to identifying the significant difference in the three operational categories defined by the IR. They are different but require and follow an interdependent regulatory logic and system to execute the certification and assessment of the conformity of the unmanned aerial systems. These different legal philosophies tried to make it possible to establish a uniform set of rules for the whole of the European Union, which is proportionate to the risks arising from their use and size, and which is capable of handling their differences with conventional aircraft. Further clarification is required, however, in the interpretation of rules and the application thereof: the system will not work if EASA does not provide proper guidance on problematic issues to avoid fragmentation of the single market of professional drones.

\section{ACKNOWLEDGEMENT}

The work was realized based on Agreement No. NKFIH-866-5/2020, with the support of the National Research, Development and Innovation Office and the Ministry of Innovation and Technology, financed by the Security Technologies National Laboratory.

\section{AUTHOR CONTRIBUTIONS}

Zs. Sándor: Conceptualization, Writing, Review and editing.

M. Pusztai: Conceptualization, Writing, Review and editing. 


\section{DISCLOSURE STATEMENT}

The authors declare that they have no known competing financial interests or personal relationships that could have appeared to influence the work reported in this paper.

\section{REFERENCES}

[1] Unmanned aerial vehicles market - Growth, trends, COVID-19 impact, and forecasts (2021 - 2026) 2021, [cited 05-07-2021]. https://www.mordorintelligence.com/industryreports/uav-market

[2] Europe Unmanned Aerial Vehicle (UAV) market 2020-2026 by offering, frame, MTOW, technology, range, class, end user, and country: Trend forecast and growth opportunity. Report, no. 4901755 2020, [cited 05-07-2021]. https://www.researchandmarkets.com/reports/ 4901755/europe-unmanned-aerial-vehicleuav-market-2020

[3] "Consolidated version of the treaty on the functioning of the European Union," Official Journal of the European Union, col. 47, no. C 326, pp. 1-390, 2012.

[4] "Commission delegated regulation (EU) 2019/945 of 12 March 2019 on unmanned aircraft systems and on third-country operators of unmanned aircraft systems," Official Journal of the European Union, vol. 62, no. L 152, pp. 1-40, 2019.

[5] "Commission implementing regulation (EU) 2019/947 of 24 May 2019 on the rules and procedures for the operation of unmanned aircraft", Official Journal of the European Union, vol. 62, no. L 152, pp. 45-71, 2019.

[6] "Regulation (EC) No 765/2008 of the European Parliament and of the Council of 9 July 2008 setting out the requirements for accreditation and market surveillance relating to the marketing of products," Official Journal of the European Union, vol. 51, no. L 218, pp. 30-47, 2008.

[7] "Decision No 768/2008/EC of the European Parliament and of the Council of 9 July 2008 on a common framework for the marketing of products, and repealing Council Decision 93/465/EEC," Official Journal of the European Union, vol. 51, no. L 218, pp. 82-128, 2008.

[8] ASD-STAN Standardization: Direct Remote ID - Introduction to the European UAS digital remote technical standard, 2021, [cited 05-072021].

https://asd-stan.org/wp-content/uploads/ASDSTAN_DRI_Introduction_to_the_European_d igital_RID_UAS_Standard.pdf

[9] European Commission, Notified Bodies NANDO, [cited 05-07-2021]. https://ec.europa.eu/growth/tools-

\section{ORCID}

Zs. Sándor http://orcid.org/0000-0001-7117-9069

M. Pusztai http://orcid.org/0000-0001-6983-4554

databases/nando/index.cfm?fuseaction=directi ve.main

[10] Good practice for market surveillance. [cited 05-07-2021].

https://ec.europa.eu/docsroom/documents/230 41

[11] "Regulation (EU) 2019/1020 of the European Parliament and of the Council of 20 June 2019 on market surveillance and compliance of products and amending Directive 2004/42/EC and Regulations (EC) No 765/2008 and (EU) No 305/201," Official Journal of the European Union, vol. 62, no. L 169, pp. 1-44, 2019.

[12] EASA Flying a Drone for fun - Get to know how to become a good drone pilot. Video presentation: Are you flying a \#drone for fun and want to become a good pilot? [cited 05-072021].

https://www.youtube.com/watch?v=9aXcUMy gkAY

[13] EASA Operations in the medium risk of the specific category Video presentation: The new design verification process for authorising drone operations in the "specific" category SA, [cited 05-07-2021]. https://www.youtube.com/watch?v=69EUfd4s nzY

[14] JARUS guidelines on Specific Operations Risk Assessment (SORA), Joint Authorities for Rulemaking of Unmanned Systems. JARDEL-WG6-D.04, 2019.

[15] Acceptable Means of Compliance (AMC) and Guidance Material (GM) to Commission Implementing Regulation (EU) 2019/947. European Union Aviation Safety Agency, no. 1, pp. 11-113, 2019.

[16] EASA Webinar on Design Verification and update of EASA activities: Drone design verification in the medium risk specific category operations. EASA Drones team 16 April 2021, [cited 05-07-2021]. https://www.enac.gov.it/sites/default/files/alle gati/2021-

Apr/2021_04\%20Webinar_design_verificatio n_and_update_of_EASA_activities.pdf

[17] Guidelines on Design verification of UAS operated in the 'specific' category and classified in SAIL III and IV, European Union Aviation Safety Agency, no. 1, pp. 1-7, 2021.

[18] Special condition light USA, European Union Aviation Safety Agency, no. 1, pp. 1-25, 2020. 
[19] "Commission implementing regulation (EU) 2012/748 of 3 August 2012 laying down implementing rules for the airworthiness and environmental certification of aircraft and related products, parts and appliances, as well as for the certification of design and production organizations", Official Journal of the European Union, vol. 55, no. L 224, pp. 1-92, 2012.

(c) (i) $\$$ This article is an open access article distributed under the terms and conditions of the Creative Commons Attribution NonCommercial ( $C C B Y-N C$ 4.0) license. 